\title{
Dimensões da satisfação dos usuários da Estratégia Saúde da Família
}

\author{
Components of user satisfaction of the Family Health Strategy
}

Artigo Original | Original Article

Laelson Rochelle Milanês Sousa (1a), Rafael Fernandes de Mesquita (2b), Fátima Regina Ney Matos, PhD (3b), Luana Kelle Batista Moura, PhD (4a), Maria Eliete Batista Moura, PhD (5a),

\author{
(1) Doutorando em Ciências pelo Programada de Pós-Graduação em Enfermagem Fundamental da Escola de Enfermagem de Ribeirão Preto. Universidade de São Paulo - São Paulo - Brasil. \\ (2) Doutorando do programa de Pós-Graduação em Administração de Empresas da Universidade Potiguar - UnP - Rio Grande do Norte - Brasil. \\ (3) Doutora em Administração de Empresas. Docente do Instituto Superior Miguel Torga - Coimbra - Portugal e da Universidade Potiguar - UnP. \\ (4) Doutora em Odontologia. Docente do Centro Universitário UNINOVAFAPI - Piauí - Brasil. \\ (5) Doutora em Enfermagem. Docente do Programa de Pós-Graduação em Enfermagem da Universidade Federal do Piauí - Piauí - Brasil. \\ (a) Colaboraram na concepção do estudo, revisão teórica, análise e interpretação dos dados, redação do artigo e aprovação da versão final. \\ (b) Colaboraram com as análises estatísticas, revisão crítica relevante do conteúdo intelectual do trabalho, ajustes na redação e aprovação da versão final.
}

Autor para correspondência | Corresponding author: Laelson Rochelle Milanês Sousa; E-mail: laelson_@hotmail.com

\section{Palavras-Chave}

Saúde da Família Atenção Primária à Saúde Qualidade da Assistência à Saúde Satisfação do Paciente Análise Multivariada

\section{Keywords}

Family Health

Primary Health Care

Quality of Health Care

Patient Satisfaction

Multivariate analysis

\section{RESUMO}

Objetivo: O objetivo foi analisar as variáveis explicativas da satisfação da qualidade de serviços da Estratégia Saúde da Família.

Método: Estudo quantitativo realizado com 353 usuários da Estratégia Saúde da Família, atendidos na zona leste do município de Teresina (PI, Brasil). Utilizou-se a escala SERVQUAL, alimentada por um instrumento adaptado, com frases afirmativas e analisados por meio de técnicas multivariadas: análise fatorial e regressão múltipla.

Resultados: As análises multivariadas possibilitaram a redução da escala utilizada para cinco dimensões da qualidade de serviços prestadas, que responderam pela explicação de $99,7 \%$ da variação do índice geral calculado e utilizado no modelo de regressão múltipla. Todos os pressupostos de ambas as técnicas foram respeitados.

Conclusão: A confiabilidade foi a dimensão preponderante na percepção da qualidade de serviços e o modelo testado foi validado, tornando-se possíveis suas réplicas em outros contextos empíricos e a ampliação de seu escopo.

\section{ABSTRACT}

Objective: The aim was to analyze the explanatory variables of satisfaction of the service quality of the Family Health Strategy.

Method: A quantitative study with 353 users of the Family Health Strategy, attended in the eastern zone of the city of Teresina (PI, Brazil). The SERVQUAL scale, filled by an adapted instrument, was used with affirmative sentences and analyzed using multivariate techniques: factorial analysis and multiple regression.

Results: The multivariate analysis allowed the reduction of the scale used for five dimensions of the quality of services provided, which accounted for the explanation of $99.7 \%$ of the variation of the general index calculated and employed in the multiple regression model. All the assumptions of both techniques were respected.

Conclusion: Reliability was the preponderant dimension in the perception of the quality of services and the tested model was validated, making possible its replicas in other empirical contexts and the expansion of its scope.

Nota das editoras: o artigo encontra-se redigido em Português do Brasil. 


\section{INTRODUÇÃO}

A preocupação com a satisfação dos usuários dos serviços de atenção primária à saúde tem sido constantemente referenciada na literatura acadêmica especializada internacional (Cimas et al., 2016). Tanto trabalhos que utilizam escalas já conhecidas e validadas, quanto estudos que desenvolvem e validam novos instrumentos têm dado atenção à satisfação de usuários de serviços de saúde por se constituir um elemento fundamental na assistência centrada no paciente (Mohammadi e Mohammadi, 2012; Silberman, MorenoAltamirano, Hernández-Montoya, Martínez-González, e Díaz-López, 2016; Qin, Prybutok e Prybutok, 2016).

O modelo SERVQUAL (Parasuraman, Zeithaml e Berry, 1985) é dos mais utilizados quando se busca avaliar a qualidade de serviços a partir da percepção de seus usuários, sem restrições de tipologia organizacional, e a Estratégia Saúde da Família (ESF) - anteriormente denominada Programa Saúde da Família (PSF) - já foi alvo de estudos que verificavam a satisfação de seus usuários por meio do SERVQUAL (Dias, Vieira, Rios e Ramos, 2011a).

A ESF busca reorganizar os serviços de Atenção Primária no país, que era focada numa lógica biomédica. Apesar de suas intenções, há muitos óbices de cunho político, uso de poder e de distribuição de recursos financeiros, que dificultam sua efetividade, tornando-a objeto de constantes avaliações de qualidade de serviços prestados. O uso de diversos instrumentos acadêmicos pode ser útil neste quadro de análise a apoiar a tomada de decisão quanto ao desenvolvimento da ESF (Oliveira e Pereira, 2013; Penna e Queiróz, 2015; Dias, Lopes, Vieira e Ramos, 2011b).

O Service Quality ou modelo/escala SERVQUAL, medição para qualidade de serviço, foi desenvolvido a partir de um modelo de verificação da satisfação do cliente, que é função da diferença entre as expectativas e o desempenho da organização (Oliver, 1980). Esta era sua intenção analítica e seu uso inicial, mas diversos estudos têm utilizado a escala para avaliar a qualidade de serviços prestados por organizações, por meio de métodos descritivos e de análises univariadas em contexto nacional (Dias et al., 2011b; Penna e Queiróz, 2015).

Em contexto internacional, a situação é complementada por análises fatoriais e demais técnicas multivariadas, justificando-se o uso destas modalidades analíticas no cenário brasileiro, que permanece à margem de estudos que aproveitam este modelo em análises descritivas ( $\mathrm{Li}$ et al., 2015). Tais estudos não verificam a consistência interna da escala, os fatores ou dimensões que se formam a partir das correlações entre as variáveis, e nem a relevância de cada uma destas na explicação da variação de um índice geral da qualidade de serviços ( $L i$ et al., 2015; Purcărea, Gheorghe e Petrescu, 2013), o que se traduz em um gap analítico de uso da escala.

Neste sentido, há relevância em manter em foco a avaliação da qualidade dos serviços prestados por instituições públicas na Atenção Primária à saúde, especialmente a ESF, gerando um conhecimento que possa balizar as ações e o desenvolvimento de novos programas e estratégias. Os resultados de avaliações constantes também podem melhorar programas já existentes, pois avaliar a qualidade dos serviços por meio da satisfação dos usuários é uma forma eficiente na identificação de falhas e contribui para a melhoria da qualidade dos serviços (Almeida e Brito, 2014; Ogunnowo, Olufunlayo e Sule, 2015). Desta forma, o estudo objetivou analisar as variáveis explicativas da satisfação da qualidade de serviços da Estratégia Saúde da Família.

\section{MÉTODO}

Esta é uma pesquisa de abordagem quantitativa e descritiva, que utilizou análise multivariada de dados obtidos por meio da aplicação de instrumento de coleta adaptado. O questionário aplicado foi estruturado utilizando frases afirmativas relacionadas à percepção de satisfação dos usuários de serviços da ESF do posto do Uninovapafi.

As afirmações correspondem às cinco dimensões da qualidade, conforme modelo SERVQUAL com poucas adaptações para esta pesquisa (inclusão do nome do posto de saúde em substituição ao termos genéricos empresa ou organização), também utilizado em outros estudos mais recentes (Dias et al., 2011a, 2011b). As dimensões investigadas correspondem à confiabilidade (Variáveis P1 a P5), presteza (P6 a P9), segurança (P10 a P13), empatia (P14 a P18) e tangibilidade (P19 a P22). As opções de resposta são graduadas em escala Likert, para medir o nível de concordância em relação às afirmações, variando de "concordo totalmente" a "discordo totalmente", com 5 pontos.

A pesquisa foi realizada com usuários atendidos por duas equipes em um mesmo posto da ESF na zona leste da cidade de Teresina, no estado do Piauí, Brasil. No momento da pesquisa, 927 famílias eram beneficiadas pelas duas equipes que compunham a ESF investigada. Foram seguidos os seguintes critérios de inclusão de sujeitos no estudo: estar cadastrado na ESF do local selecionado; ter idade igual ou superior a 18 anos; ser capaz de responder ao questionário; e ter sido atendido no mínimo uma vez na ESF. O critério de exclusão utilizado era ser funcionário da ESF ou de qualquer outro serviço 
ligado à Fundação Municipal de Saúde de Teresina. Para estimar a população de indivíduos atendidos, foi utilizada a média de pessoas por família em Teresina, calculada conforme dados do último censo realizado pelo Instituto Brasileiro de Geografia e Estatística (IBGE), realizado em 2010 (média de 3,65 pessoas) com estimativa de 3.384 pessoas atendidas (IBGE, 2010). Estes dados apoiaram o cálculo para determinação do tamanho da amostra, que guiou a quantidade de questionários aplicados e, para isso, utilizou-se fórmula apresentada na Equação 1.

\section{Equação 1}

$$
\begin{aligned}
& n=\frac{Z^{2} \times P \times Q \times N}{e^{2} \times(N-1)+Z^{2} \times P \times Q} \\
& \text { Com: } \\
& Z=\text { nível de confiança (95\%); } \\
& P=\text { quantidade de acerto esperado (50\%); } \\
& Q=\text { quantidade de erro esperado (50\%); } \\
& N=\text { população (3.384 pessoas); } \\
& e=\text { nível de precisão (5\%); } \\
& n=345 .
\end{aligned}
$$

Optou-se por realizar a coleta de dados nas próprias residências dos usuários, pois, durante o pré-teste do questionário feito em uma ESF de outra região da cidade, os pesquisadores identificaram que os usuários se sentiam constrangidos ao responder as entrevistas na Unidade Básica de Saúde. A amostra contou com 353 participantes, oito indivíduos além do número indicado no cálculo amostral, ou seja, uma margem que possibilitou substituir questionários incompletos ou incorretos (Mesquita, Sousa, Martins e Matos, 2014).

Os dados foram tabulados e analisados com apoio do software estatístico Statistical Package for the Social Sciences (SPSS), versão 20.0. Para a análise, foram realizados procedimentos que identificavam: (i) estatísticas descritivas para dados sociodemográficos; (ii) análise de confiabilidade da escala; e (iii) técnicas analíticas multivariadas, com análise fatorial e regressão linear múltipla. Logo após, foi utilizado o teste do alfa de Cronbach, para verificar a consistência interna da escala utilizada, e foram efetuados procedimentos de análise fatorial exploratória (AFE), para identificar as dimensões subjacentes à correlação entre as variáveis adotadas para o estudo. $O$ teste de regressão linear múltipla utilizou um índice como variável dependente e os fatores encontrados na análise anterior, como independentes na equação, possibilitando análise de suas participações na explicação do Índice de Satisfação da Qualidade de Serviços da ESF (SQS).

A pesquisa foi submetida ao Comitê de Ética e Pesquisa do Centro Universitário UNINOVAFAPI, sendo aprovada, sob parecer $n^{\circ} 893.065$ e CAAE $n^{\circ}$ 38532614.0.0000.5210. Os usuários que aceitaram participar do estudo assinaram o Termo de Consentimento
Livre e Esclarecido. Foi garantido o anonimato de todos os participantes da pesquisa, pois os questionários não foram identificados.

\section{RESULTADOS}

A amostra selecionada possuía o seguinte perfil: 253 $(71,7 \%)$ do sexo feminino; maioria com idades entre 21 e 30 anos (29,5\%) e entre 31 e 40 anos (30,9\%); estado civil solteiro (33,7\%) e casado (46,7\%); e a escolaridade era Ensino Médio incompleto (36,8\%) e completo (43,3\%); a renda familiar de $64,1 \%$ da amostra variava entre um e dois salários mínimos; e 58,9\% destes indivíduos não estavam empregados à época da coleta de dados.

A confiabilidade da escala de 22 itens adaptada para este estudo foi verificada a partir do alfa de Cronbach, por meio de modelo de consistência interna baseada em correlações, aqui com valor de 0,922, ou seja, acima do considerado mínimo ideal $(0,7)$ (Hair, Black, Babin, Anderson e Tatham, 2009). Para proceder com a AFE, primeiro foram verificados seus pressupostos e a conveniência de sua aplicação. A matriz de correlação entre todas as variáveis foi gerada e avaliada, demonstrando relações altas e significativas $(p<0,01)$.

O segundo conjunto de teste foi constituído por Kaiser-Meyer-Olkin (KMO) e pelo teste de esfericidade de Bartlett, utilizando-se todas as variáveis da escala, com resultados apresentados na Tabela 1.

\section{Tabela 1}

Teste Kaiser-Meyer-Olkin (KMO) e o teste de esfericidade de Bartlett - Escala SERVQUAL

\begin{tabular}{lcc}
\hline Medida de adequação de amostra KMO & 0,890 \\
& $\chi^{2}$ aproximado & 4085,085 \\
Teste de esfericidade de Bartlett & Grau de liberdade & 231 \\
& Significância & $<0,001$ \\
\hline
\end{tabular}

Nota. $\chi^{2}=$ Qui-quadrado.

A significância do teste de esfericidade de Bartlett rejeitou a hipótese nula de que a matriz de correlação é uma matriz identidade, situação adequada para a AFE. O KMO também apresentou resultado adequado. Deste modo, a proposta de análise foi possível e conveniente. No entanto, esta primeira solução com todas as variáveis inclusas apresentou algumas inconsistências na associação com os fatores, pois, após extração e rotação, duas variáveis possuíam cargas fatoriais em duas dimensões com valores próximos e abaixo de 0,6 considerado inadequado (Hair et al., 2009). Foram elas a P6 ("Funcionários da Estratégia Saúde da Família da 
localidade dizem exatamente quando os serviços de saúde serão executados") e a P18 ("Os funcionários da Estratégia de Saúde da Família da localidade compreendem suas necessidades específicas de saúde”). Estas variáveis foram excluídas da análise.

A escala final utilizada para o estudo possuía 20 variáveis e cinco fatores identificados na AFE.
Para essa solução, foi calculado um novo KMO $(0,880)$ e foi realizado um novo teste de esfericidade de Bartlett $(p<0,001)$, indicando que não houve prejuízo na demonstração de utilidade da AFE. A Tabela 2 apresenta as cargas fatoriais, comunalidades $\left(H^{2}\right)$ e porcentual de variância explicado acumulado por cada um dos fatores. Os fatores foram nomeados a partir de inspiração nas dimensões originais do modelo SERVQUAL.

Tabela 2

Análise Fatorial Exploratória (AFE) - Escala SERVQUAL

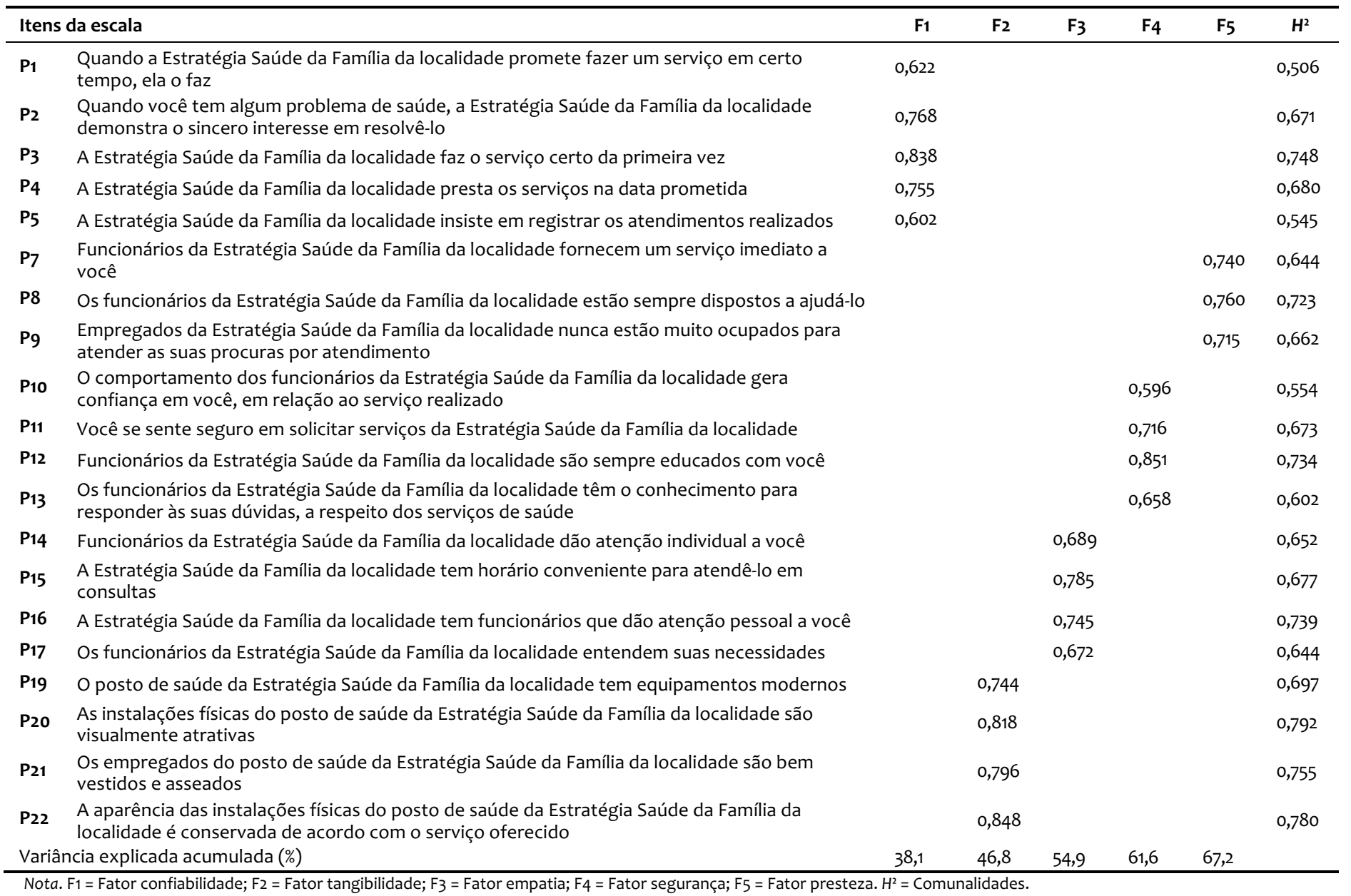

Após reduzir a escala de 22 itens aos cinco fatores expostos na Tabela 2, procedeu-se a uma análise de regressão múltipla, tendo por variável dependente o SQS, calculado a partir da média simples dos valores de todas as variáveis da escala. As variáveis independentes da equação foram os cinco fatores, conforme Equação 2.

Equação 2

$\mathrm{SQS}=\beta_{0}+\beta_{1} \mathrm{~F} 1+\beta_{2} \mathrm{~F} 2+\beta_{3} \mathrm{~F} 3+\beta_{4} \mathrm{~F} 4+\varepsilon$

(Modelo)
O modelo de regressão apresentou coeficiente de determinação $\left(R^{2}\right)$ de $99,7 \%$ e coeficiente de determinação ajustado (adjusted R square) de 99,4\%. Esses altos valores indicaram o quanto as variáveis independentes utilizadas explicaram a variação do item dependente, o SQF, fenômeno observado. O modelo, como um todo, possuía significância estatística $(p<0,001)$, como se verifica na Tabela 3, bem como todas as variáveis independentes em relação ao modelo ( $p<0,001)$, conforme pode ser observado na Tabela 4. 
Tabela 3

Sumária do Modelo de Regressão

\begin{tabular}{|c|c|c|c|c|c|c|c|c|c|}
\hline \multirow{2}{*}{$R$} & \multirow{2}{*}{$R^{2}$} & \multirow{2}{*}{$\mathrm{R}^{2}$ ajustado } & \multirow{2}{*}{ Erro padrão } & \multicolumn{5}{|c|}{ Variações estatísticas } & \multirow{2}{*}{ Durbin-Watson } \\
\hline & & & & Variação do $\left(R^{2}\right)$ & F modificado & $d f_{1}$ & $d f 2$ & Sig. $F$ modificado & \\
\hline $0,997^{*}$ & 0,994 & 0,994 & 0,06917 & 0,994 & 10809,738 & 5 & 347 & $<0,001$ & 1,901 \\
\hline
\end{tabular}

Nota. *Preditores (constantes): confiabilidade, tangibilidade, empatia, segurança, presteza; † variável dependente: Índice de Satisfação da Qualidade dos Serviços da Estratégia Saúde da Família.

Tabela 4

Coeficientes Análise de Regressão

\begin{tabular}{|c|c|c|c|c|c|c|c|}
\hline \multirow{2}{*}{ Modelo } & \multicolumn{2}{|c|}{ Coeficientes não padronizados } & \multirow{2}{*}{$\begin{array}{c}\text { Coeficientes padronizados } \\
\text { Beta }\end{array}$} & \multirow{2}{*}{$t$} & \multirow{2}{*}{$p$} & \multicolumn{2}{|c|}{ Tolerância da estatística colinear } \\
\hline & B & Erro padrão & & & & Tolerância & VIF \\
\hline (Constante) & 3,358 & 0,004 & & 912,213 & $<0,001$ & & \\
\hline Confiabilidade & 0,435 & 0,004 & 0,506 & 117,934 & $<0,001$ & 1,00 & 1,00 \\
\hline Tangibilidade & 0,396 & 0,004 & 0,460 & 107,337 & $<0,001$ & 1,00 & 1,00 \\
\hline Empatia & 0,383 & 0,004 & 0,446 & 104,016 & $<0,001$ & 1,00 & 1,00 \\
\hline Segurança & 0,337 & 0,004 & 0,392 & 91,487 & $<0,001$ & 1,00 & 1,00 \\
\hline Presteza & 0,358 & 0,004 & 0,416 & 97,107 & $<0,001$ & 1,00 & 1,00 \\
\hline
\end{tabular}

Nota. Fator de variância inflacionário. Variável dependente: Índice de Satisfação da Qualidade dos Serviços da Estratégia Saúde da Família.

O teste de regressão múltipla apresentou a soma dos quadrados no valor de 258,615 , com resíduos no valor de 1,660. O valor da soma dos quadrados foi o resíduo quadrado, caso fosse utilizada apenas a média da variável dependente SQS para predição. No modelo de regressão, ao incluir as variáveis independentes na equação, esse resíduo apresentou o valor de 1,160, ou seja, diminuiu e melhorou o poder de predição do modelo. $O$ teste de Análise de Variância (ANOVA) $(F=10809,738 ; p<0,001)$ com significância estatística atestou que 0 modelo foi válido ao rejeitar a hipótese de que $O R^{2}=0$. Estes resultados são visíveis na Tabela 5.

\section{Tabela 5}

Análise de Variância

\begin{tabular}{|c|c|c|c|c|c|}
\hline Modelo & Soma dos quadrados & Graus de liberdade & Média do quadrado & $F$ & Significância \\
\hline Regressão & 258,615 & 5 & 51,723 & $10.809,738$ & $<0,001$ \\
\hline Resíduos & 1,660 & 347 & 0,005 & & \\
\hline Total & 260,276 & 352 & & & \\
\hline
\end{tabular}

Nota. Preditores: (constante), confiabilidade, tangibilidade, empatia, segurança, presteza Variável dependente: Satisfação da Qualidade dos Serviços da Estratégia Saúde da Família.

A Tabela 4 apresenta os coeficientes B da equação, os coeficientes padronizados beta e os valores de $\mathrm{p}$ para significância estatística de cada uma das variáveis na equação. Todas as variáveis incluídas na equação apresentaram relação significante $(p<0,01)$. Deste modo, a equação do modelo de regressão linear foi expressa conforme a Equação 3.

\section{Equação 3}

$\mathrm{SQS}=3,358+0,435 \mathrm{~F} 1+0,396 \mathrm{~F}_{2}+0,383 \mathrm{~F}_{3}+0,337 \mathrm{~F}_{4}+0,358 \mathrm{~F}_{5}$
Os pressupostos para a análise de regressão linear foram analisados e respeitados (autocorrelação serial, multicolinearidade, normalidade dos resíduos e homocedasticidade). A ausência de autocorrelação serial foi verificada pelo teste de Durbin-Watson, que indicou valores adequados quando abaixo de 2. $\mathrm{Na}$ Tabela 3, o valor de 1,901 para o teste Durbin-Watson foi considerado apropriado. A multicolinearidade foi pressuposta ausente, pois as variáveis independentes dessa equação de regressão foram fatores encontrados na AFE, mas o teste de Fator de Variância Inflacionário e as estatísticas de colinearidade, indicadas na Tabela 3, apoiaram essa afirmação. Fator de Variância 
Inflacionário abaixo de 10 e tolerância até 1 foram indicativos de ausência de colinearidade ou de valores aceitáveis. O teste Pesarán-Pesarán indicou a homocedasticidade dos resíduos, pois a hipótese nula, que afirmou esta situação, não foi rejeitada $(p=0,196)$. A normalidade dos resíduos foi verificada no teste Kolmogorov-Smirnov, não rejeitando a hipótese nula que afirmou serem normais os resíduos $(p=0,519)$.

A análise dos pressupostos da regressão linear múltipla atestou a validade e a adequação do modelo e de seus resultados.

\section{DISCUSSÃO}

Avaliar a satisfação de usuários de serviços de saúde é um processo complexo que engloba múltiplas dimensões. O modelo SERVQUAL mostrou-se adequado para avaliação da satisfação dos usuários da ESF, pois dispõe de cinco dimensões abrangentes que englobam desde atendimento individual a instalações e estrutura física: Confiabilidade, Tangibilidade, Empatia, Segurança e Presteza (Parasuraman et al., 1985).

A Tangibilidade considera as instalações físicas do ambiente, bem como equipamentos e demais condições tangíveis. Confiabilidade relaciona-se à capacidade das equipes de oferecerem serviços com confiança e exatidão, desenvolvendo confiança. A Presteza, por outras vezes denominada "responsabilidade", está para a disposição a ajudar dos prestadores de serviço. Segurança relaciona-se à capacidade para esclarecer dúvidas, à transmissão de confiança e à manutenção de sigilo sobre determinados assuntos. A Empatia corresponde ao interesse e à atenção individual (Parasuraman et al., 1985; Dias et al., 2011a, 2011b).

Os fatores encontrados na análise fatorial foram nomeados a partir de inspiração nas dimensões originais da escala, pois o modelo exploratório identificou fatores semelhantes àqueles propostos nos estudos prévios, corroborando estes resultados e ampliando o escopo e as possibilidades de utilização da escala.

O SERVQUAL é empregado em diversas realidades e culturas. Em avaliação da qualidade de serviços de saúde em nove cidades da China, por meio do SERVQUAL, o estudo apontou que as dimensões Empatia e Confiabilidade foram as mais relevantes na percepção da qualidade dos serviços (Li et al., 2015). Esta pesquisa corrobora os resultados encontrados, ao demonstrar a confiabilidade como fator preponderante, tanto na AFE quanto no modelo de regressão. A aplicação do instrumento mostrou-se relevante, ao indicar pontos estratégicos para a melhoria na qualidade do serviço.
Na Nigéria, o uso do SERVQUAL indicou lacunas nos serviços de saúde como o tempo de espera por atendimento (Ogunnowo et al., 2015). No contexto do Sistema Único de Saúde, uma das principais lacunas do serviço e que gera insatisfação de seus usuários, é a marcação de consultas para especialidades médicas que demanda muito tempo de espera e resulta em longas filas (Szwarcwald, Damacena, Souza Júnior, Almeida e Malta, 2016). Entretanto, não é uma realidade exclusiva do Brasil. Problema semelhante é encontrado nos serviços de saúde pública da Indonésia onde existe insatisfação dos usuários pela morosidade no atendimento (Handayani, Hidayanto, Sandhyaduhita, Kasiyah, e Ayuningtyas, 2015).

Em outra aplicação do SERVQUAL na busca para identificação do grau de satisfação, na Arábia Saudita, a dimensão Confiabilidade foi destaque (Al-Momani, 2015). Dados semelhantes foram encontrados em estudos nacionais na ESF (Moreira, Reis, Bizarria e Barbosa, 2015). Tratando a confiabilidade como a dimensão de maior destaque, as pesquisas identificam insatisfações quanto ao atendimento das expectativas dos usuários.

Um dos pontos de insatisfação dos usuários de serviços de alta complexidade do Serviço Único de Saúde Brasileiro - SUS é a pontualidade dos funcionários, porém usuários cardíacos deram ênfase à dimensão da segurança (Borges, Carvalho e Silva, 2010). Presteza e segurança foram citadas como aquelas dimensões de maior influência na percepção da qualidade dos serviços prestados. Este resultado, apesar de diferir do que foi encontrado aqui, demonstra que os cenários não podem ser generalizados, implicando o aprofundamento de condições que acarretam observação de uma ou outra dimensão como principal.

Elevados níveis de satisfação de usuários dos serviços de saúde, de forma geral, têm relações significativas com a qualidade da saúde das pessoas. Durante o planejamento e implementação de políticas de saúde deve ser levado em consideração à melhoria da satisfação dos usuários (Cimas et al. 2016). Desta forma, o modelo SERVQUAL possibilita a identificação de lacunas na prestação de serviços que podem ser passíveis de preenchimento após análise gerencial da situação, ampliando a percepção de qualidade de serviços e satisfação do consumidor (Purcărea, Gheorghe e Petrescu, 2013). A presente pesquisa mostrou-se eficaz na identificação das possíveis lacunas existentes na prestação de serviços de saúde em atenção básica, com a identificação das dimensões da satisfação que receberam menor avaliação e classificação.

\section{CONCLUSÃO}


A partir de análises descritivas e multivariadas, encontraram-se cinco fatores, com a preponderância identificada da confiabilidade, semelhantes às dimensões originais da escala SERVQUAL, que foram utilizados como variáveis independentes na análise de regressão múltipla, cuja variável dependente foi a Satisfação da Qualidade de Serviços da Estratégia Saúde da Família. Dessa forma, foi possível verificar a influência dessas dimensões identificadas na explicação da variação do índice calculado, observando a ordem de relevância de cada uma e sua participação na equação do modelo de regressão. $O$ estudo obteve altos coeficientes de determinação, utilizando fatores obtidos a partir de uma análise significativa e com alto Kaiser-Meyer-Olkin. Todos os pressupostos das duas técnicas multivariadas foram respeitados, validando o modelo e suas interpretações.

As contribuições deste trabalho se estendem à ampliação do uso da escala, que já é vastamente utilizada em diversos contextos, especialmente em estudos de comportamento do consumidor, e aqui tratou do usuário de serviços públicos de saúde. Buscar a satisfação do usuário da Estratégia Saúde da Família torna-se outra importante consideração deste estudo, enquanto contribui para despertar interesse de autoridades em melhorias nos serviços prestados por programas desse tipo, indicando dimensões essenciais para a compreensão da percepção de qualidade de serviços prestados.

Apesar do pouco percentual restante para explicação, além daquele observado no modelo de análise estatística, considera-se que há muito de aspectos subjetivos a serem investigados em análises sociais desse fenômeno. Tais aspectos ficaram evidentes durante a aplicação dos questionários, escapando ao escopo do estudo, e podem indicar futuras pesquisas na área: delongas em marcações de consulta para especialidades médicas; ausência do agente comunitário de saúde, que deveria ser figura periódica na região e nas casas; questões de trato dos atendentes e subjetividades inerentes às condições socioeconômicas do local de atendimento do posto da Estratégia Saúde da Família e seu entorno. Essa lista de observações pode se tornar um problema de pesquisa que auxilie na compreensão do objeto investigado neste trabalho e contribua para ampliação do escopo deste estudo.

Conflito de interesses | Conflict of interest: nenhum | none.

Fontes de financiamento | Funding sources: CAPES - Fundação Coordenação de Aperfeiçoamento de Pessoal de Nível Superior.

\section{REFERÊNCIAS}

Almeida, F. A. e Brito, F. A. (2014). Planejamento e avaliação de programas de saúde: contribuições do Modelo RE-AIM para Enfermagem [Planning and evaluation of health programs: contributions of the RE-AIM Model for Nursing]. Revista LatinoAmericana de Enfermagem, 22(4), 527-528. doi:101590/0104

Al-Momani, M. M. (2015). Gap analysis between perceptions and expectations of medical-surgical patients in a public hospital in Saudi Arabia. Medical Principles and Practice, 25(1), 79-84. doi:10.1159/000441000

Borges, J. B. C., Carvalho, S. M. R. D. e Silva, M. A. D. M. (2010). Quality of service provided to heart surgery patients of the Unified Health System-SUS. Brazilian Journal of Cardiovascular Surgery, 25(2), 172-182. doi:10.1590/S0102-76382010000200008

Cimas, M., Ayala, A., García-Pérez, S., Sarria-Santamera, A. e João Forjaz, M. (2016). The patient satisfaction questionnaire of EUprimecare project: measurement properties. International Journal for Quality in Health Care, 28(3), 275-280. doi:10.1093/intobc/mzwo24

Dias, O. V., Vieira, M. A., Dias, J. P. e Ramos, L. H. (2011a). The dimensions of user satisfaction of the family health program: trust and empathy. Acta Paulista de Enfermagem, 24(2), 225-231. doi:10.1590/S0103-21002011000200011

Dias, O. V., Lopes, J. A. S., Vieira, M. A. e Ramos, L. H. (2011b). Tangibilidade no Programa Saúde da Família: Percepção dos usuários [Tangibility in the Family Health Program: Perception of the users]. Revista de Atenção Primária à Saúde, 14(3), 327-335.

IBGE (2010). Fundação Instituto Brasileiro de Geografia e Estatística. Censo Demográfico de 2010. Fundação Instituto Brasileiro de Geografia e Estatística, dados referentes à cidade de Teresina - Piauí. Recuperado de http://cidades.ibge.gov.br/xtras/perfil.php?lang=\&codmun=221100\&searc $\mathrm{h}=$ piaui|teresina

Handayani, P. W., Hidayanto, A. N., Sandhyaduhita, P. I., Kasiyah, J. e Ayuningtyas, D. (2015). Strategic hospital services quality analysis in Indonesia. Expert Systems with Applications, 42(6), 3067-3078. doi:10.1016/j.eswa.2014.11.069

Hair, J. F., Black, W. C., Babin, B. J., Anderson, R. E. e Tatham, R. L. (2009). Análise multivariada de dados. Bookman Editora.

Li, M., Lowrie, D. B., Huang, C. Y., Lu, X. C., Zhu, Y. C., Wu, X. H., ... Zhao, P. (2015). Evaluating patients' perception of service quality at hospitals in nine Chinese cities by use of the ServQual scale. Asian Pacific Journal of Tropical Biomedicine, 5(6), 497-504. doi:10.1016/j.apjtb.2015.02.003

Mesquita, R. F., de Sousa, M. B., Martins, T. B. e Matos, F. R. N. (2014). Óbices metodológicos da prática de pesquisa nas ciências administrativas. Revista Pensamento Contemporâneo em Administração, 8(1), 50-65. doi:10.12712/rpca.v8i1.387

Mohammadi, A. e Mohammadi, J. (2012). Evaluating quality of health services in health centres of Zanjan district of Iran. Indian Journal of Public Health, 56(4), 308. doi:10.4103/0019-557X.106422

Moreira, R. N., Reis, D. T. C., Bizarria, F. P. A. e Barbosa, F. L. S. (2015). Qualidade de serviços: análise de uma unidade do programa saúde da familia [Quality of services: analysis of a unit of the family health program]. Gestão e Saúde, 6(2), 1267-1288. doi: 10.18673/gs.v6i2.22468

Ogunnowo, B. E., Olufunlayo, T. F. e Sule, S. S. (2015). Client perception of service quality at the outpatient clinics of a general hospital in Lagos, Nigeria. Pan African Medical Journal, 22, 68. doi:10.11604/pamj.2015.22.68.6228

Oliver, R. L. (1980). A cognitive model of the antecedents and consequences of satisfaction decisions. Journal of Marketing Research, 17(4), 460-469. doi:10.2307/310499

Oliveira, M. A. D. C. e Pereira, I. C. (2013). Primary health care essential attributes and the family health strategy. Revista Brasileira de Enfermagem, 66(Spec), 158-164. doi:10.1590/S0034-71672013000700020 
Parasuraman, A., Zeithaml, V. A. e Berry, L. L. (1985). A conceptual model of service quality and its implications for future research. The Journal of Marketing, 49(4), 41-50. doi:10.2307/1251430

Penna, C. M. D. M. e Queiróz, E. D. S. (2015). Conceitos e práticas de enfermeiros no trabalho com famílias [Conceptions and practices of nurses working with families]. Texto \& Contexto - Enfermagem, 24(4), 941-949. doi:10.1590/0104-0707201500001500014

Purcărea, V. L., Gheorghe, I. R. e Petrescu, C. M. (2013). The assessment of perceived service quality of public health care services in Romania using the SERVQUAL scale. Procedia Economics and Finance, 6, 573585. doi:10.1016/S2212-5671(13)00175-5

Qin, H., Prybutok, V. e Prybutok, G. (2016). Quantitative comparison of measurements of urgent care service quality. Health Marketing Quarterly, 33(1), 59-77. doi:10.1080/07359683.2016.1131581
Silberman, M. S., Moreno-Altamirano, L., Hernández-Montoya, D., Martínez-González, A. e Díaz-López, H. O. (2016). Construcción y validación de un instrumento para medir la satisfacción de los pacientes del primer nivel de atención médica en la Ciudad de México [Construction and validation of an instrument to measure the satisfaction of patients at the first level of medical care in Mexico City]. Gaceta Médica de México, 152, 43-50.

Szwarcwald, C. L., Damacena, G. N., Souza Júnior, P. R. B. D., Almeida, W. D. S. e Malta, D. C. (2016). Percepção da população brasileira sobre a assistência prestada pelo médico. Brasil, 2013 [Perception of the Brazilian population on medical health care. Brazil, 2013]. Ciência \& Saúde Coletiva,21(2), 339-350. doi:10.1590/141381232015212.19332015 